\title{
Activity with Signs and Speaking About It: Exploring Students' Mathematical Lines of Thought Regarding the Derivative
}

\author{
Annika M. Wille ${ }^{1}$ D
}

Received: 28 March 2019 / Accepted: 21 September 2019 / Published online: 10 December 2019

(C) The Author(s) 2019

\begin{abstract}
In order to participate proficiently in mathematical activity, a student needs to become fluent in both mathematical sign activity and speaking about the activity with signs. From the theoretical viewpoint of the philosophers Wittgenstein and Peirce, on the one hand, mathematics is seen as a sign game, while on the other hand experiments with signs allow for observable, communicable and describable reasoning. In this article, the focus is on the student's sign activity and his or her speaking about it and the question of how they are intertwined. To that end, a method is presented to display the interplay of student's activity with signs and speaking about it, and in particular, to reconstruct the student's line of argumentation. This is applied to a case study where imaginary dialogues written by a class of grade 11 students on the topic "derivative" were analyzed.
\end{abstract}

Keywords Calculus · Language and mathematics · Peirce $\cdot$ Semiotics $\cdot$ Wittgenstein

\section{Introduction}

Sign activity and speaking about sign activity are both part of mathematical activity. They are intertwined in various ways. It is precisely this linkage that is the focus of this article by means of examples of students' explanations and reasoning regarding the derivative.

In the literature, the role of mathematical signs, such as symbols, terms, equations and graphs, often is described as a means for mathematical activity, for example to serve as representations for abstract mathematical objects (e.g. Duval, 2006). In this article, mathematical signs will be considered from the perspective of Wittgenstein and Peirce (cf. Dörfler, 2016), where sign activity is seen as a central and inevitable

Annika M. Wille

annika.wille@aau.at

1 Institut für Didaktik der Mathematik, Universität Klagenfurt, Sterneckstraße 15, 9010, Klagenfurt, Austria 
part of mathematical activity. Furthermore, the meaning of these signs arises from the use of the signs, thus, largely from activity with the signs. Therefore, these signs are not only a means but are objects of mathematical activity as well. Additionally, speaking about sign activity is also inevitable within mathematical reasoning. The ways in which mathematical activity, sign activity and speaking about sign activity intertwine will be elaborated upon below. From this perspective, in order to participate more and fully in mathematical social practice, a learner needs to become fluent in mathematical sign activity and in addition this sign activity needs to be linked to speaking about the sign activity. Neither one nor the other can be dispensed with. In complex subjects, as with the topic of "derivative", sign activity and speaking about it are linked to each other in various ways. Therefore, how a student uses mathematical signs in an explanation on this topic is particularly interesting.

In some countries, calculus is only taught at college or university. In other countries, like Austria, it is already part of the school curriculum in grades 11 and 12. Students exhibit various difficulties in learning calculus (e.g. Ferrini-Mundy \& Graham 1991; Orton, 1983; Rasmussen et al. 2014). In particular, studies mention that one difficulty in understanding the derivative is that various other terms need to have been learned beforehand, such as "function", "limit", and "difference quotient" (e.g. Park 2015; Thompson 1994a; Weigand 2014; Wille 2017b; Zandieh 2000).

This article is divided into two parts: In the first part Wittgenstein's view of mathematics is presented and referenced to Peirce's theory of signs with an emphasis on connections between mathematical sign activity and speaking about this activity. Afterwards, these considerations are related to the subject area of "derivative", which leads to specific research questions regarding:

1. The connections a student establishes between mathematical signs, between verbal terms, or between signs and terms (see RQ 1).

2. The process of establishing these connections in an explanation (see RQ 2).

The second part presents an analysis method together with a study which was carried out in a grade 11 school class and takes up the research questions stated at the end of the theoretical part. The analysis of each student's mathematical communication is presented in a visualization that maps the student's mathematical lines of thought that are displayed in their communication. In particular, there is a focus on how a student intertwines sign activity and speaking about the activity. In that way, differences between the high-, average- and low-achieving students in this class can be determined and learning difficulties can be explained.

\section{Theoretical Framework}

\section{Mathematics in the Light of Wittgenstein and Peirce}

\section{Wittgenstein: Mathematics as a Sign Game}

In the view of the Austrian philosopher Ludwig Wittgenstein (1889-1951) mathematics is a kind of sign game or language game where the meaning of a mathematical 
sign, symbol, word or proposition arises from its use within the game (cf. Wittgenstein, PI, RFM, WWK). He compares mathematics in this regard with the game of chess, where, for example, a certain figure does not have a meaning outside the game, but where the meaning is obtained by the rules that hold for the figure, for example, how to move it (cf. Wrigley, 1977, p. 53). Thus, as a chess figure can be moved according to the game rules, signs in mathematics can be operated with or derived of others due to the rules in mathematics (cf. WWK, pp. 103-105). Nevertheless, Wittgenstein does not see mathematics purely as a game. In contrast to a pure game, Wittgenstein emphasizes that mathematics obtains its legitimation from the "use outside mathematics" (cf. RFM, p. 133e). Nevertheless, "the meaning of the signs, symbols, and diagrams does not come from the outside of mathematics but is created by a great variety of activities with the signs within mathematics" (Dörfler, 2016, p. 27). From this viewpoint, understanding and learning mathematics means to progressively learn the rules of the game and how they are connected, hence, how rules can be derived of others. As a chess player needs to practice the game in order to become fluent in playing and to understand the game, a mathematics learner needs to act with signs in order to understand mathematics.

\section{Peirce's Diagrams as Objects and Means of Mathematical Thinking}

The theory of signs of the American mathematician, logician, and philosopher Charles Sanders Peirce (1839-1914) and in particular his notions of diagram and diagrammatic reasoning can be used to characterize the activity with signs in mathematics more precisely. However, it is neither the aim of this article, nor can it be done in a few pages, to extensively describe Peirce's theory. Elaborations in greater detail can be found, for example, in Hoffmann (2005a, b, 2007); Dörfler (2005, 2006, 2016), and Otte (2011).

It is important to note that the term diagram used by Peirce differs from the common understanding of the notion of a diagram in mathematics and mathematics education. A diagram, in Peirce's sense, does not have a mandatory geometric connotation. Examples of diagrams in mathematics are algebraic terms or equations, function graphs, and also geometric figures or Hasse diagrams of lattices that are usually termed diagrams in the mathematical language. But, it should also be mentioned that not every visualization that is used in mathematics or mathematics education is a diagram in Peirce's sense.

Diagrams are based on inscriptions, for example on paper, and have several characteristics that qualify them as a diagram (cf. Dörfler, 2016, pp. 25-26). One of them is that they belong to a system of representations that specifies how to construct diagrams and how to experiment with them. In the following, this type of activity with diagrams given by a system of representations is referred to as diagrammatic activity. Furthermore, diagrams are complex signs: indices can direct the attention to something, they form a subset of icons and represent relations because of conventions. Therefore, a diagram also has a symbolic character (cp. Hoffmann, 2005b, p. 46). In particular, a diagram is not a diagram by itself. But a sign can be interpreted as diagram, if an individual knows a system of representations through which it can be constructed and above all that offers possibilities of operations on this diagram, 
whereby operations are understood in a broad sense. For example, a mathematical term can be interpreted as a diagram if an individual knows the conventionalized rules about how to construct it and how to experiment with it. But for a different individual, who does not know a corresponding representational system, that same term may appear to be just an artistic pattern. In this way, a verbal term can also be a mathematical diagram if there are such possibilities of operations.

For Peirce, mathematical reasoning is diagrammatic reasoning that consists of constructing, experimenting, observing, noting, and assuring (cf. Peirce, NEM IV: pp. 47-48; Hoffmann, 2007, p. 3). Thus, diagrams are not only the means of the generation of knowledge, but also objects. Hoffmann (2007) describes "mathematical reasoning as a process in which an individual (or a group of individuals) constructs an external representation, and experiments with this representation playfully and creatively, in order to clarify, structure, and coordinate thinking processes" (ibid., p. 21). Thus, the experiments with diagrams are not only algorithmic manipulations, but involve creativity as a part of a process to coordinate thinking processes.

This fits well with Wittgenstein's view that the meaning of a mathematical sign arises from its use within the sign game. The rules on how to experiment with diagrams given by a system of representations can be seen as a part of the sign game rules. And as one can see how a chess figure is moved, the activity on diagrammatic inscriptions can be perceived with physical senses. As a consequence, this enables communication about the activity with signs. The role of communication, in particular, to speak about the activity with diagrams, will be elaborated below.

\section{Diagrammatical Activity and Speaking About It}

In the theoretical view outlined above, diagrammatical activity is seen as an inevitable part of mathematical activity. Furthermore, to speak about this activity is in the same way indispensable in mathematics. In the following, the connections between and intertwining of these two activities are outlined.

\section{Denotations}

The first connection regards denotations for different diagrams, in particular, diagrams that belong to different representational systems. In mathematics, often the same denotation is used if one sees a kind of correspondence between the representational systems along with their diagrams. For example, the word "function" is used as a denotation for different diagrams, that is, for a function term as well as for a function graph. Thus it is possible to speak about the properties of these denotations as for example: "If a function is differentiable at a point, it is continuous at this point."

Instead of seeing a term and a graph as different representations of an abstract mathematical object "function", from the diagrammatical point of view, one starts with systems of representations and their diagrams and if there is a kind of 
correspondence a denotation emerges. ${ }^{1}$ Even if there is a correspondence between diagrams of different representational systems, it is still possible that different representational systems allow different diagrammatic activities of an individual. Since a diagram represents relations, different relations can be the focus of the observer for diagrams in different systems of representations. This can lead to different experiments with the diagrams in order to solve a problem.

Still, it can be helpful to use the same denotation for diagrams of different representational systems, since that way one can talk about properties of the "entities". In particular, the words for properties allow for connections to be made between verbal terms and diagrammatic activity. However, it could be problematic if a learner, who is not fluent enough in diagrammatic activity, learns a mathematical term mainly by speaking about diagrams but with only little diagram use, and who therefore connects the denotation insufficiently with the corresponding diagrams and how they are operated with. In the study presented in this article, examples will be shown where students use language detached from diagrammatic activity. This provides an explanation for the learning difficulties observed (cf. e.g. the case of Peter below).

\section{Interpretations of Diagrammatic Reasoning}

A second connection between diagrammatic activity and speaking about it is outlined in Dörfler (2005) regarding interpretations of diagrammatic reasoning. As described above, language can be used to talk about "the results of (observing) the diagrammatic operations and deduce on the conceptual level new properties of the latter" (Dörfler 2005, p. 59). Dörfler calls this an "economic substitute" (ibid., p. 59), which is the case if the complexity of diagrammatic inscriptions and the experiments with them causes the use of verbal terms. Furthermore, Dörfler describes different reasons, where limitations of diagrammatic reasoning can cause the use of speaking about diagrams.

One limitation has to do with reasoning about infinity or rather reasoning about all diagrams of a certain class. One example concerns natural numbers. The inscription $n$ can be considered as a diagram constructed by the system of representation that contains the Peano axioms as rules. Thus, $n$ can be called a natural number as a denotation of this diagram. Furthermore, by these axioms (which can be regarded as rules in the sign game according to Wittgenstein) it follows that a diagrammatic inscription $n+1$ can be constructed. Until now the reasoning is diagrammatic. But, to conclude that there are infinitely many natural numbers, is, according to Dörfler, an interpretation of diagrammatic reasoning. " $\mathbb{N}$ has no diagram", as Dörfler states (ibid., p. 62). Analogous considerations can be made for the existence of infinitely many prime numbers or the set of all real numbers.

Interestingly, in this case, there is not only a move from diagrammatic reasoning to speaking about diagrammatic activity, but also the other way around. Because of the interpretation "there are infinitely many natural numbers", one invents the sign $\mathbb{N}$

\footnotetext{
${ }^{1}$ Instead of correspondence, for example Thompson (1994b) used the term "sense of invariance" that may be produced by "representational activities".
} 
and likewise $\mathbb{Q}$ or $\mathbb{R}$ or $\mathbb{C}$ for all rational, real or complex numbers. Therefore, in the diagrammatic "world" one can continue to operate with these signs and experiment with them, as for example when using the phrase "for all $\varepsilon>0$ with $\varepsilon \in \mathbb{R}^{\text {". }}$

Here, one can see how diagrammatic sign activity and speaking about it intertwine in mathematics: Language is not only used to verbalize experiences with diagrams, but also to interpret diagrammatic reasoning with a return to the "world of mathematical diagrams". Thus, interpretations of diagrammatic reasoning can lead to a diagrammatization as a backflow from "speaking about" to diagrammatic activity.

This has consequences for the learning process. Diagrammatic activity can be observed directly, but interpretations are not likewise accessible to our senses as experiments on diagrams. That might be a reason why students have problems in cases when not only diagrammatic reasoning but also interpretations and their reflow as diagrams is the focus. That is in accordance with what a university student of mine, a preservice teacher, wrote in a text: "Intuitive understanding is not a fan of infinity" (translated by the author). ${ }^{2}$ An example is the equation $0 . \overline{9}=1$. Problems with this equation are reported in various articles (e.g. Eisenman, 2008; Monaghan, 2001; Tall \& Schwarzenberger, 1978). Furthermore, in Müller-Hill and Wille (2018) it was shown, that there are students who prove the equation but nevertheless state that $0 . \overline{9}$ is less than 1 . To conclude the equality the definition of limit is necessary, where the phrase "for all $\varepsilon>0$ " is central. ${ }^{3}$ Now, "for all" can only be understood when there is a set of all real numbers which is an interpretation of diagrammatic reasoning. Thus, an individual needs to accept the interpretation and not only (which is difficult enough) the rules of how to act with diagrams and their implications, in order to participate in the mathematical "game". Likewise below, in the findings of the study presented here, problems arise in cases where students try to explain what the limit is (cf. e.g. the case of Katharina and Maria below). In the following, the focus turns to the diagrammatical sign activity and speaking about it regarding the derivative.

\section{Diagrammatical Sign Activity and Speaking About It Regarding the Derivative}

\section{Connecting Terms and Diagrams}

On the level of speaking about diagrammatic sign activity, verbal terms can be connected in the use of language. These terms can be denotations or interpretations of diagrammatic reasoning. Regarding the topic of "derivative", these verbal terms are not mathematical diagrams themselves, since there are no operations on them given by a representational system. One can only speak about operations on diagrams for which these verbal terms are denotations. Furthermore, a verbal term can arise from applications of diagrams where diagrams are used as models in outer-mathematical contexts. Figure 1 (cf. similar tables in Wille (2017b, p. 1397) and in Danckwerts and Vogel (2006, p. 57 \& p. 85)) shows various verbal terms that are used regarding

\footnotetext{
${ }^{2}$ The original text in German was: "Das intuitive Verständnis hält nichts von der Unendlichkeit."

${ }^{3}$ Even if the formula for the infinite sum of the geometric series is used in order to conclude $0 . \overline{9}=1$, the definition of limit is necessary to prove this formula.
} 


\begin{tabular}{|c|c|c|c|c|c|}
\hline $\begin{array}{l}\text { algebraic- } \\
\text { analytical }\end{array}$ & $\begin{array}{l}\text { function or value of } \\
\text { a function }\end{array}$ & $\begin{array}{l}\text { difference of func- } \\
\text { tion values }\end{array}$ & difference quotient & limit & $\begin{array}{l}\text { differential quo- } \\
\text { tient or derivative }\end{array}$ \\
\hline $\begin{array}{l}\text { geometric- } \\
\text { graphical }\end{array}$ & $\begin{array}{l}\text { graph or point on } \\
\text { a graph }\end{array}$ & $\begin{array}{l}\text { difference of the } \\
\text { value } y \text { of points }\end{array}$ & secant slope & $\begin{array}{l}\text { geometric descrip- } \\
\text { tion of an approach } \\
\text { of two points }\end{array}$ & tangent slope \\
\hline $\begin{array}{l}\text { application- } \\
\text { based }\end{array}$ & $\begin{array}{l}\text { according to the } \\
\text { specific applica- } \\
\text { tion, e.g., distance } \\
\text { at a point of time }\end{array}$ & $\begin{array}{l}\text { according to the } \\
\text { specific applica- } \\
\text { tion, e.g., absolute } \\
\text { difference of dis- } \\
\text { tances }\end{array}$ & $\begin{array}{l}\text { rate of change or } \\
\text { relative change, } \\
\text { e.g., average speed }\end{array}$ & $\begin{array}{l}\text { according to the } \\
\text { specific applica- } \\
\text { tion, e.g., descrip- } \\
\text { tion of an approach } \\
\text { of two points of } \\
\text { time }\end{array}$ & $\begin{array}{l}\text { local rate of change } \\
\text { or momentary rate } \\
\text { of change, e.g., mo- } \\
\text { mentary speed }\end{array}$ \\
\hline
\end{tabular}

Fig. 1 Use of verbal terms regarding the topic "derivative"

the topic "derivative". They are differentiated in algebraic-analytical, geometricgraphical, and application-based terms. In what follows, I distinguish horizontal and vertical connections according to this table.

On the level of diagrammatic activity, the correspondence between function terms and function graphs is central in school mathematics, ${ }^{4}$ where these diagrams belong to different representational systems. In what follows, the representational system to which diagrams like function terms belong will be termed algebraic-analytical and the representational system to which diagrams like function graphs belong will be termed geometric-graphical. Furthermore, the terms horizontal and vertical, which refer to the table in Fig. 1, are transferred to diagrams: A vertical connection represents a correspondence of two diagrams in different representational systems, here, between the algebraic-analytical and the geometric-graphical representational system. A horizontal connection corresponds to a diagrammatic line of reasoning within one representational system, possibly using diagrammatizations of interpretations.

In the algebraic-analytical system of representations the (horizontal) diagrammatic line of reasoning to derive the differential quotient uses diagrams of the form:

$$
f(x), f(x)-f(y), \frac{f(x+h)-f(x)}{h} \text { and } f^{\prime}(x)=\lim _{h \rightarrow 0} \frac{f(x+h)-f(x)}{h} .
$$

Of course, instead of these diagrams, it is possible to write other diagrams due to manipulation rules of the representational system, like, for example, $\lim _{x \rightarrow x_{0}} \frac{f(x)-f\left(x_{0}\right)}{x-x_{0}}$ instead of $\lim _{h \rightarrow 0} \frac{f(x+h)-f(x)}{h}$ or using $g(t)$ instead of $f(x)$.

As argued before the step from $\frac{f(x+h)-f(x)}{h}$ to $\lim _{h \rightarrow 0} \frac{f(x+h)-f(x)}{h}$ requires a reflow from speaking about diagrams, because of the phrase "for all $\varepsilon>0$ with $\varepsilon \in \mathbb{R}$ " in the definition of the limit. Hence, a precondition of following this step is to accept the interpretation that there is an infinite set of real numbers.

Due to the geometric-graphical system of representations, secants and tangents can be constructed and one can reason about their slope, for example understood as the angle of the gradient triangle. Here, a horizontal connection uses diagrams like graphs, secant lines and their slopes and tangent lines together with their slopes.

\footnotetext{
${ }^{4}$ Of course, this correspondence also plays a role in university mathematics, which however is not the focus here.
} 


\section{Research Questions Concerning the Connections Between Diagrammatic Activity and Speaking About It}

As discussed, an individual can establish diagrammatically horizontal and vertical connections regarding the derivative and communicate about it. With the perspective of seeing diagrammatic reasoning as a central and inevitable part of mathematical activity, the question arises of how when speaking about connections of mathematical verbal terms, a learner links his or her discourse to diagrams and diagrammatic activity.

Clearly, someone who is experienced in diagrammatic activity regarding the derivative, thus, who links horizontally and vertically diagrams according to one or several representational systems, may use denotations to speak about these experiences. However, if a learner speaks about mathematical verbal terms as if they were entities, what the learner says will not necessarily be based on their experience with diagrams. Instead, the learner could have memorized the "speaking about" detached from diagrammatic activity. One can imagine, for example, that a student could not follow the diagrammatic activity that was presented and discussed in class, and that he or she therefore learned non-diagrammatic phrases by heart.

Taking these considerations into account, the above question can be specified as follows as the first research question for the study presented here:

(RQ 1) How does a student horizontally and vertically connect verbal terms and diagrams regarding the topic "derivative"? In particular, in which way does he or she use diagrams when speaking about this topic?

Because of the significance of the connection of the diagrams

$$
\frac{f(x+h)-f(x)}{h} \text { and } \lim _{h \rightarrow 0} \frac{f(x+h)-f(x)}{h},
$$

respectively the verbal terms "difference quotient" and "differential quotient", the question arises not only whether a student connects them, but how this connection is integrated into the student's line of argumentation. This leads to the second research question:

(RQ 2) Which lines of thought can be observed when a student acts with diagrams regarding the topic "derivative" and/or speaks about the denotations "difference quotient" and "differential quotient" or "derivative"?

\section{Method}

\section{A Method to Explore a Student's Thinking Processes Regarding the Derivative}

The following requirements guided the decision on which form of communication was chosen for this study: In order to address the research questions, it should be possible for the communicating student to combine the writing of diagrams and speaking about diagram activity. One possibility for such a combination is to use written language. In that way, even in one sentence, a student can reason with diagrams and 
about diagrams. Since there are pronounced differences between oral and written speech (e.g. Vygotsky, 1986, p. 180), this is discussed below in relation to the form of communication utilized in this study. Furthermore, having the individual work of a student makes it easier to focus on that student. Additionally, in order to minimize the influence on the student after an initial stimulus there should be no further interruption. Finally, the form of communication should enable the student not only to talk about connections in a static way, but also in a procedural way, i.e. how connections are established and not only which.

Therefore, the method of imaginary dialogues (Wille 2008, 2017a) was chosen: An imaginary dialogue means that a student works individually to compose a written dialogue between two protagonists discussing a mathematical task or question. ${ }^{5}$ The student provides a continuation of a given initial dialogue as shown in Fig. 2. The imaginary dialogue is in written form, but its content is a conceived spoken dialogue. Thus, the imaginary dialogue possesses aspects of spoken and written language. This can be illustrated using Koch and Österreicher's (2012) model, where forms of communication are placed between two poles: language of immediacy and language of distance either as phonic or graphic realization. Here, imaginary dialogues can be positioned about midway between these two poles on the graphic side (Wille 2017a, p. 37). This means that students tend to write how they understood something rather than only what they understood (cf. ibid., p. 42).

The task in the case study presented here was an initial dialogue (see Fig. 2) that was given to grade 11 students in a class at a gymnasium in Graz, Austria, in November 2016. The students' grades in mathematics from the previous year were also known. Before this task, the students had been taught calculus including the topic of derivative by their teacher. ${ }^{6}$ Each of the 20 students in the class continued the initial dialogue as individual work. The dialogues were written in German and translated by the author.

Because of the focus on the connection "difference quotient", "differential quotient" (regarding RQ 2), the task addresses a question concerning the limit. When the function $f$ is a polynomial, the limit of the difference quotient can be calculated by first cancelling $h$ in the denominator. Then, in order to obtain the limit, it seems like one is substituting $h$ by 0 , even though, in the $\varepsilon-\delta$-definition of the limit of a function there is no such substitution. In particular, this does not work for an arbitrary $f$. However, in the case of polynomials that are mainly treated in school, substituting $h$ by 0 leads to the ostensible problem that "the denominator will become 0 " which one protagonist addresses.

Since this is a difficult task, the students were not expected to solve this issue extensively. However, the conflict that was addressed in the initial dialogue should encourage the students to reason about horizontal connections, in particular, about the step from the difference quotient to the differential quotient. That way, horizontal

\footnotetext{
${ }^{5}$ One approach that is related to imaginary dialogues is that of a lesson play (Zazkis, Liljedahl \& Sinclair, 2013), where, in contrast to imaginary dialogues, it is prospective teachers, not students, who write the dialogues.

${ }^{6}$ In Austria calculus is part of the school curriculum.
} 
Two students are talking to each other. We call them S1 and S2. Continue their dialogue.

S1: Hi! Can you explain something for me?

S2: Yes, sure.

S1: You know we discussed the derivative in class. First, we had the difference quotient

$$
\frac{f\left(x_{0}-h\right)-f\left(x_{0}\right)}{h}
$$

Then, there was the limit, whereby $h$ approaches 0 .

S2: I remember. What do you want to know?

S1: If $h$ approaches 0 , then the denominator will become 0 and nothing works anymore!

S2: Okay. I'll explain it to you clearly with an example what the derivative means. Then, it will also be clear why the limit is there and what happens when $h$ approaches 0 .

S1: Thank you very much! Because it is not clear to me, what the difference quotient and the derivative actually are.

Fig. 2 The initial dialogue that was given to each student to be continued

connections are slightly more in focus than vertical connections. An additional task that explicitly emphasizes vertical connections is referred to further research.

In order to address the research questions, the aim of the analysis of the students' imaginary dialogues is to investigate their line of reasoning. Thus, an analysis sheet (see Fig. 4, on the left) was designed for this purpose. The analysis sheet has the same columns as the table of Fig. 1. Additionally, there is one column to notate algebraic-analytical, geometric-graphical, or application-based diagram usage or language usage to speak about diagrams, abbreviated with "a.-a.", "geo.", and "appl.”. Furthermore, analysis tables (see Fig. 4, on the right) have been designed to summarize the more detailed analysis sheets. How the sheets and tables were filled in is described below and then illustrated with the help of examples.

\section{First Step of the Analysis}

The first step of the analysis for each imaginary dialogue was to fill out an analysis sheet (see Fig. 4, on the left): According to the word use, a phrase is put as a circle in one column. A circle can also denote various phrases (or sentences) in succession, but only if a single verbal term is used and not several. For example, if the question and the following response use the same term, as can be seen in row 4 in Figs. 3 and 4, these phrases are coded as one circle. Phrases such as "Ah, I understand", which link phrases about mathematics in the dialogue, were not coded in the analysis sheet as separate circles.

A circle is shaded if diagrams are used in the phrase. In cases where diagrams are used exclusively, this is notated as a shaded square. For example, the phrase "So imagine that you have a second-degree function" was coded as a non-shaded circle in the column "function/function values". The phrase "apply the difference quotient $\frac{\Delta y}{\Delta x}$ " was coded as a shaded circle in the column "difference quotient". And finally, the diagram $\frac{f(3+h)-f(3)}{h}$ was inserted as a shaded square in the column "difference quotient". All three examples were described as algebraic-analytical. 
S2: The difference quotient is the average change between two $x$ values, how it changes on average in this interval.

S1: I see, I understand.
But, what is now the derivative?

S2: The derivative of a function yields a graph, which gives the slope of the original function.

For example the derived $x^{2}$ is a linear function, a straight line, where you can read out the slope at any moment.

S1: At any moment, is that the difference quotient, too?

S2: No, that is the differential quotient, which is $\frac{d y}{d x}$. It yields the slope at any moment.

That has something to do with the limit.

From the difference quotient you can get to the differential quotient.

You can also calculate it by inserting the $x$ value of the point in the equation of the derivative function.

S1: Thank you very much! Now, I understand it much better!

S2: By the way: Within the formula $\frac{f\left(x_{0}-h\right)-f\left(x_{0}\right)}{h} x_{0}$ stands for the first $x$ value and $h$ yields the distance to the second $x$ value.

Fig. 3 Translation of Oliver's imaginary dialogue

In the analysis sheet, circles can be connected by lines, either dashed or solid: Two circles are connected by a dashed line if two terms are connected by speaking about them. For example, a sentence like "You should know, that the difference quotient is

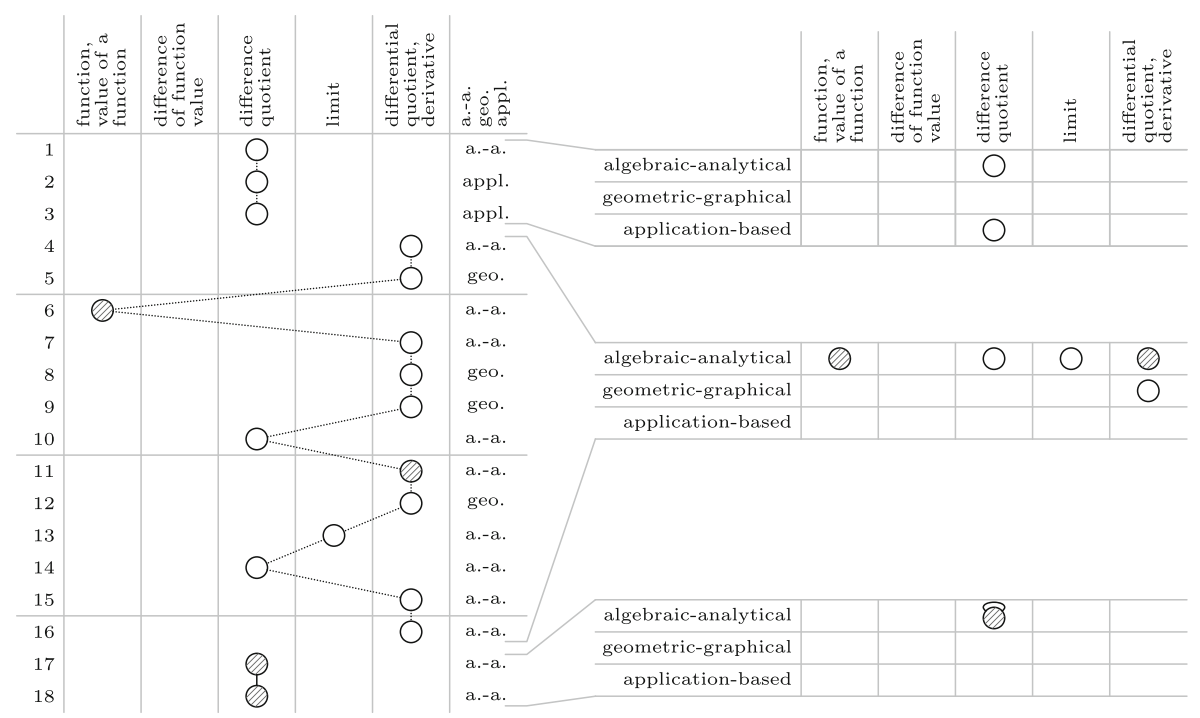

Fig. 4 The analysis sheet and analysis tables for Oliver's imaginary dialogue 
the rate of change" was denoted as two non-shaded circles in the column "difference quotient", the first labelled as algebraic-analytical, the second application-based, and both connected by a dashed line. However, two circles or squares are connected by a solid line, if two diagrams are linked diagrammatically, either horizontally by diagrammatic activity or vertically by describing the diagrammatical correspondence of an algebraic-analytical and geometric-graphical diagram. For example, the equation $\frac{4+4 h+h^{2}-4}{h}=\frac{4 h+h^{2}}{h}$ was denoted as two shaded squares in the row difference quotient and connected with a solid line.

A solid line together with a dashed line denotes a connection by diagrammatic activity and speaking about it. For example, the sentence: "And if I have for example $f(x)=x^{3}$, it will be $f^{\prime}(x)=3 x^{2}$, won't it?" was denoted as a shaded circle in the column "function/function value" and a shaded circle in the column "derivative" connected with two parallel lines, one of them solid, the other dashed. That way the line of the student's thoughts that he or she put on paper can be displayed for each imaginary dialogue.

\section{Second Step of the Analysis}

In a second step of the analysis, condensed analysis tables (see Fig. 4, on the right) were built from each analysis sheet. ${ }^{7}$ The tables can be seen as summaries of the corresponding analysis sheets, in order to see at a glance, with regard to RQ 1, the totality of the established connections.

When in an analysis sheet the circles are connected it is regarded as one line of thought. As soon as two circles are not connected, the second circle is understood as the beginning of another line of thought. For example, in his imaginary dialogue a student first talks about the difference quotient and then he writes: "Okay. Let's come to the derivative." This sentence and the sentences that follow do not refer to the previous content. Therefore, a non-shaded circle in the column of "differential quotient" is drawn without a line connecting it to the previous circle. At this point I would like to emphasize that with connected or unconnected circles only a statement is made about what appears on paper in the imagined dialogue as a line of thought.

Each analysis table now represents exactly one line of thought, as it becomes visible in the imaginary dialogue. Therefore, several analysis tables can belong to one analysis sheet.

A circle in a table field denotes that, in the corresponding line of thought within the analysis sheet, one or several circles can be found in the same column and are denoted with the same label ("a.-a", "geo.", or "appl."). For example, a shaded circle in the table field "algebraic-analytical/differential quotient" denotes that in the line of thought within the corresponding analysis sheet, there is either at least one shaded circle in the column "differential quotient" which is denoted as "algebraic-analytical", or there is at least one non-shaded circle and, in addition, at least one shaded square, both in the column "differential quotient" and denoted as "algebraic-analytical".

\footnotetext{
${ }^{7}$ The notation of the shaded and non-shaded circles in tables is inspired by a study of Zandieh (2000). However, Zandieh's circles denote something different than the study presented here.
} 
Dashed lines in the analysis sheet are not specially denoted in the analysis table, since two circles in the same table already means that they belong to one line of thought. However, solid lines in the analysis sheet are notated as solid lines in the table as well. That way, the diagrams which a student connects diagrammatically can be seen.

\section{Results}

Before the analysis sheets and tables are discussed and compared as a whole, two imaginary dialogues are presented in detail to visualize how they are reflected in the sheets and tables. In addition, a brief insight into the imaginary dialogues of three other students is given.

\section{Two Examples in Detail}

The first imaginary dialogue is written by Oliver, a low-achieving student ${ }^{8}$ and the second imaginary dialogue is written by a high-achieving student called Jakob. ${ }^{9}$

\section{Oliver's Imaginary Dialogue}

The English translation of Oliver's imaginary dialogue can be found in Fig. $3{ }^{10}$ In order to better see the single phrases that were coded, more line breaks were inserted into the text than Oliver used himself. Thereby, a numbered row in Fig. 3 visualizes to which circle in a column on the analysis sheet the phrase belongs. ${ }^{11}$ Therefore, in what follows, the term "row" is used in a broader sense than usual to correspond to one circle in the analysis sheet. Figure 4 shows the corresponding analysis sheet and tables.

Oliver starts by talking about the difference quotient and connects vertically the algebraic-analytical and applications-based terms "difference quotient" and "average change". In row 3, Oliver explains the latter in more detail. Up to this point, he does not use any diagrams, therefore the circles in the analysis sheet are not shaded.

In row 4, Oliver begins to talk about the derivative without an explicit connection to the previous part. Therefore this is coded with no connecting line between the circles and taken as a different line of thought. In rows 5 to 9, Oliver explains that the derivative is connected to the "slope of the original function" and that the derivative of $x^{2}$ is a linear function, "where you can read out the slope at any moment" (row 9). In the analysis sheet, it can be seen how Oliver's line of thought is mainly situated in the column "differential quotient, derivative", with only one jump to the column "function, value of a function", which is the only phrase so far where he

\footnotetext{
${ }^{8}$ All names in this article are in anonymous form.

${ }^{9}$ The fact that both of these imaginary dialogues are by male students has no significance for this study. They were selected because they highlight particular differences in students' imaginary dialogues.

${ }^{10}$ The imaginary dialogues were originally written in German and translated by the author.

${ }^{11}$ Similarly, Staats (2008) represents repetitions in transcripts in order to analyze them.
} 
uses a diagram. According to the table in Fig. 1, Oliver mainly connects denotations vertically that refer to algebraic-analytical and geometric-graphical representational systems, but only with sporadic diagram use. He writes the first isolated diagram after one jump to the "function, value of a function" column without operating diagrammatically with it. Instead he explains the outcome of an operation (i.e. deriving a function) with the words "linear function".

With rows 9, 10, and 11, Oliver connects verbal terms horizontally in the column "differential quotient, derivative" and "difference quotient", again using a diagram without acting on it: Oliver's diagram use in row 11 is similar to the diagram use in row 6: he writes down a diagram without further experimenting with it. After this, he connects once again vertically algebraic-analytical in row 11 with geometricgraphical denotations in row 12.

Now, starting from rows 13 to 16, Oliver moves to the term "limit" and says vaguely: "That has something to do with the limit" (row 13). Oliver does not use diagrams or diagrammatic activity regarding the limit. Moreover, in the further course of the imaginary dialogue limit is not taken up again. Instead, he describes a horizontal connection of the terms "difference quotient" and "differential quotient" with the sentence: "From the difference quotient you can get to the differential quotient" (rows 14 and 15), but he does not specify this transition, neither diagrammatically nor by speaking about diagrammatic activities. In row 16 , there is a diagram, $x$, that is part of the diagram of the derivative, but since it is not the whole diagram, the corresponding circle is denoted non-shaded.

At the end, rows 17 and 18 are disconnected from the part above. How this part relates to what came before is not explicitly discussed, thus, there is no line connect-

ing row 16 and row 17. In row 17, Oliver talks about the diagram $\frac{f\left(x_{0}+h\right)-f\left(x_{0}\right)}{h}$ and explains the meaning of $x_{0}$ and $h$. It ends with the only diagrammatic operation in this imaginary dialogue, in which Oliver substitutes $h$ for $\Delta x$ in row 18 .

Regarding RQ 2, overall on Oliver's analysis sheet, it can be seen that he often connects denotations vertically. Furthermore, if he changes the column, he does so in rather wide jumps. Only in the lower middle part there are smaller horizontal steps, which also mention the limit (in row 13), but with no diagrammatic activity. In particular, the connections in row 13 to 15 are not from left to right, to see how to get to the differential quotient, but the other way around.

The three corresponding analysis tables (see Fig. 4, on the right) visualize Oliver's horizontal and vertical connections: The first table corresponds to rows 1 to 3 , the second to rows 4 to 16 , and the third to rows 17 and 18. Regarding RQ 1 , it can be seen that Oliver only rarely uses diagrams and there are no diagrammatic activities concerning horizontal connection. Oliver's vertical connections are only non-diagrammatical: Although he writes an algebraic-analytical diagram for the derivative, as can be seen in the second analysis table, a diagram in the geometricgraphical representation system is not used.

\section{Jakob's Imaginary Dialogue}

In contrast, Jakob is a high-achieving student. His imaginary dialogue and the corresponding analysis sheet and table can be seen in Fig. 5. 
$1 \quad$ S2: Let's look at this example: $f(x)=x^{2}+4$

S1: Okay, and what do we do with it, now?

2 S2: We calculate the difference quotient. For that, we substitute:

$$
\frac{(x+h)^{2}+4-\left(x^{2}+4\right)}{h}
$$

Now we can multiply everything out

$$
\frac{x^{2}+2 x h+h^{2}+1-x^{2}-4}{h}
$$

If we factor out and cancel, we can remove the $h$ in the denominator.

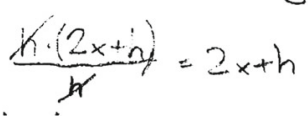

That way the problem of dividing by 0 is solved.

Now, if the $x$ value changes by $h$, we can calculate how the function values change on average in the interval $[x ; x+h]$.

8 S1: Okay. Thus, this is the difference quotient?

S2: Exactly.

9 S1: And the differential quotient?

10 S2: Well, if the change of the $x$ values (that means $h$ ) becomes steadily smaller and approaches zero,

11 then the interval in which we calculate the average rate becomes steadily smaller.

12 Therefore, if we now assume that the $x$ values do not change at all, thus, $h$ is zero,

13 we can calculate, with the help of what we calculated before, for each $x$ value the average rate exactly at this point.

14 Therefore, $2 x+0$ is the function of the derivative

15 that can be used to calculate the average rate for just every $x$ value.

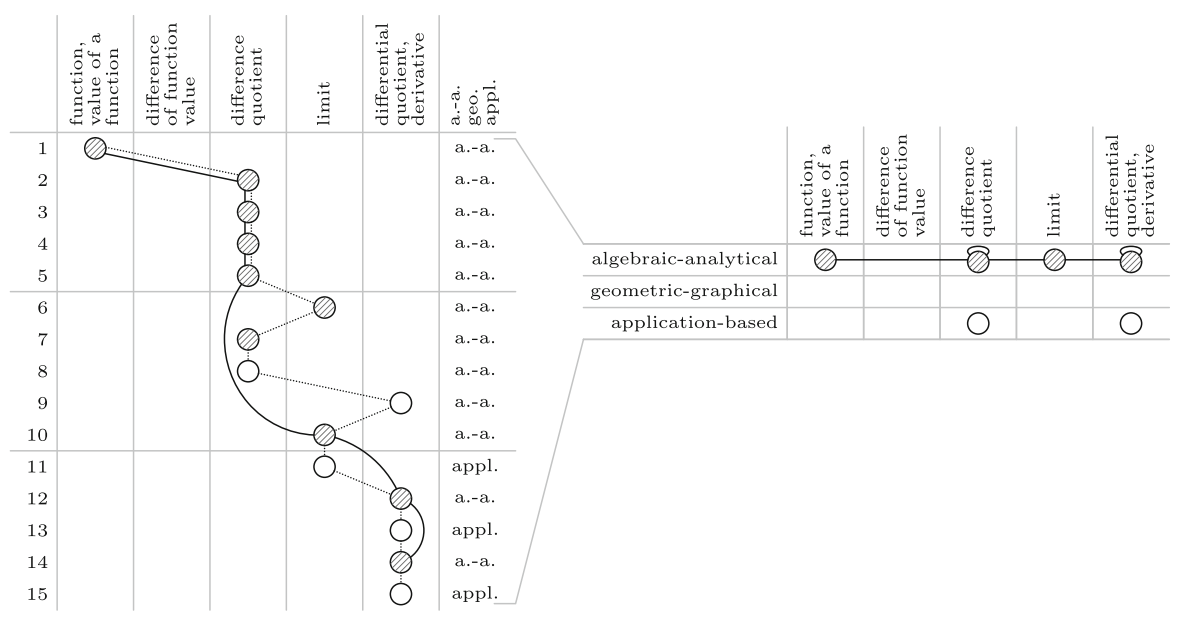

Fig. 5 Translation of Jakob's imaginary dialogue and the corresponding analysis sheet and analysis table 
Even a glance at Jakob's analysis sheet reveals great differences between his and Oliver's imaginary dialogue regarding RQ 2. One can see a movement, starting in the column "function, value of a function" via the columns "difference quotient" and "limit" to the column "differential quotient, derivative". Furthermore, the shaded circles and the solid connections display a line of diagrammatic activity. All the circles are connected. There are no breaks between the different lines of thought that can be read in the imaginary dialogue.

Looking at Jakob's imaginary dialogue in more detail, it can be seen that he starts with a concrete example $f(x)=x^{2}+4$, thus, a diagram, and transforms the corresponding difference quotient until he can cancel $h$ in the denominator (rows 4 and 5). In row 6 Jakob states that the problem $h=0$ that was mentioned in the initial dialogue in connection with the limit has now been solved. The following rows explain with the help of diagrammatic operations, why, in Jakob's view, the problem actually is solved. In particular, the diagrams in rows 5, 10, 12, and 14 are connected by diagrammatic activity. Moreover, in the middle (rows 8 and 9), there is a short excursion in which Jakob clarifies the terms "difference quotient" and "differential quotient" and at the end, in rows 11, 13, and 15, he vertically connects application-bazterms with algebraic-analytical denotations that he uses otherwise.

The analysis table in Fig. 5 (on the right) displays Jakob's horizontal and vertical connections. It becomes apparent, regarding RQ 1, that Jakob mainly argues within the algebraic-analytical system of representations. In particular, all circles in the "algebraic-analytical" row are shaded and connected to each other with solid lines. This means that Jakob not only speaks about the steps from a function to its derivative, but also uses diagrams at every point, which are connected by diagrammatic activities. Only the difference of function values is not mentioned explicitly, since he moves directly from a concrete function to the corresponding difference quotient. In comparison, vertical connections are only made to application-based terms at two columns.

Thus, it can be said that Jakob acts with diagrams in order to connect all significant (horizontal) steps from a function to its derivative within the algebraicanalytical system of representations. Vertical connections are only rarely and non-diagrammatically made.

\section{A Comparison of Oliver's and Jakob's Imaginary Dialogues}

Comparing Oliver's and Jakob's imaginary dialogues and their corresponding analysis sheets and tables, two main differences become apparent:

The first is their use (or non-use) of diagrams when moving between different "places" (i.e. columns in the analysis sheets and tables) concerning how to get to the derivative. Jakob demonstrates by example, by using the diagram $f(x)=x^{2}+4$, how the diagram $\frac{(x+h)^{2}+4-\left(x^{2}+4\right)}{h}$ is built and how with diagrammatic operations one can reach the diagram $2 x+0$ that he terms "function of the derivative". That way, he can even address the ostensible problem of the division by 0 when $h$ in the denominator approaches 0 . In contrast, Oliver writes diagrams rarely and does not operate with diagrams, except in one point when substituting $h$ by $\Delta x$. In particular, the 
derivative of $f(x)=x^{2}$ is named a "linear function" without writing the corresponding diagram (row 7). Moreover, the ostensible problem of the division by 0 is not addressed.

The second is the difference in the length of the steps between the columns. While Jakob takes small steps, and there is an overall movement from the column "function, value of a function" to the column "differential quotient, derivative", Oliver more often remains vertical in a column and then jumps across several columns. Furthermore, Jakob's imagined dialogue seems like a coherent line of thought, whereas Oliver's dialogue shows breaks, which are then reflected in several tables per line of thought. Such differences are also apparent, when the analysis sheets and tables of the entire class are considered.

\section{Further Insights in the Students' Imaginary Dialogues}

The following examples will provide insight into how a student vertically connects diagrams and how he speaks about the connection between functions and their derivative while omitting diagram activity. In addition, examples are given where students have difficulty talking about the limit.

\section{Peter's Vertical and Horizontal Connections}

Peter is an average-achieving student. His imaginary dialogue and the corresponding analysis sheet and analysis tables can be seen in Fig. 6. On the one hand, he vertically connects diagrams by explaining their correspondence in different representational systems (rows 1 to 8 ). On the other hand, he discusses horizontal connections only between functions and their derivatives, but without the steps in between and especially without any diagrammatic activity. For Peter, the connection between a function and its derivative is only a kind of "relationship", where "square functions become linear" (rows 9 to 16). This reads as if the square function disappears and (magically?) becomes a linear function. Furthermore, Peter writes that this relationship can be recognized regarding special points of the functions without explaining why. In particular, Peter compares the two diagrams $f(x)$ and $f^{\prime}(x)$ by speaking about them, but in the whole imaginary dialogue there is no explanation how the differential quotient is derived from a function by diagrammatic activity, neither in the algebraic-analytical representational system nor in the geometricgraphical.

This can also be seen in Peter's analysis sheet (see Fig. 6): First, he stays in the "difference quotient" column including two solid lines (between rows 3 and 4 and rows 6 and 7). After a split up he jumps non-diagrammatically between the column "function, value of a function", and the column "differential quotient, derivative" without a connection to the first part. Thus, Peter only refers to the diagrams themselves, but not to the diagrammatic activity that links diagrams belonging to different columns. In particular, he compares diagrams without referring to their diagrammatical connections. 
S2: So first, the difference quotient.

You can use it to calculate the average slope in a certain section in a function.

S1: You simply calculate $\frac{\Delta x}{\Delta y}$

so $\frac{y_{1}-y_{0}}{x_{1}-x_{0}}$.

S1: And why does this result in the average slope?

S2: Imagine a triangle in the graph between the dots. Something like that:

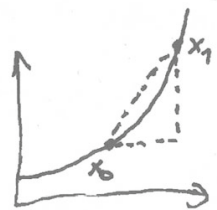

By calculating $\frac{\Delta x}{\Delta y}$

you get the average slope in the range $x_{0}$ to $x_{1}$ by the law of tangents.

S2: The derivative

is actually only an alternative to the differential quotient

to get from $f(x)$

to a related function $\left[f^{\prime}(x)\right]$. HM, difficult to explain...

S1: HEY! That's not possible! You're here to explain it to me!

S2: All right... So: During derivation,

for example, square functions

become linear and so...

You can recognize the "relationship" of the two functions by the fact that their intercepts, local extrema, inflection points or global extrema are similar to each other.

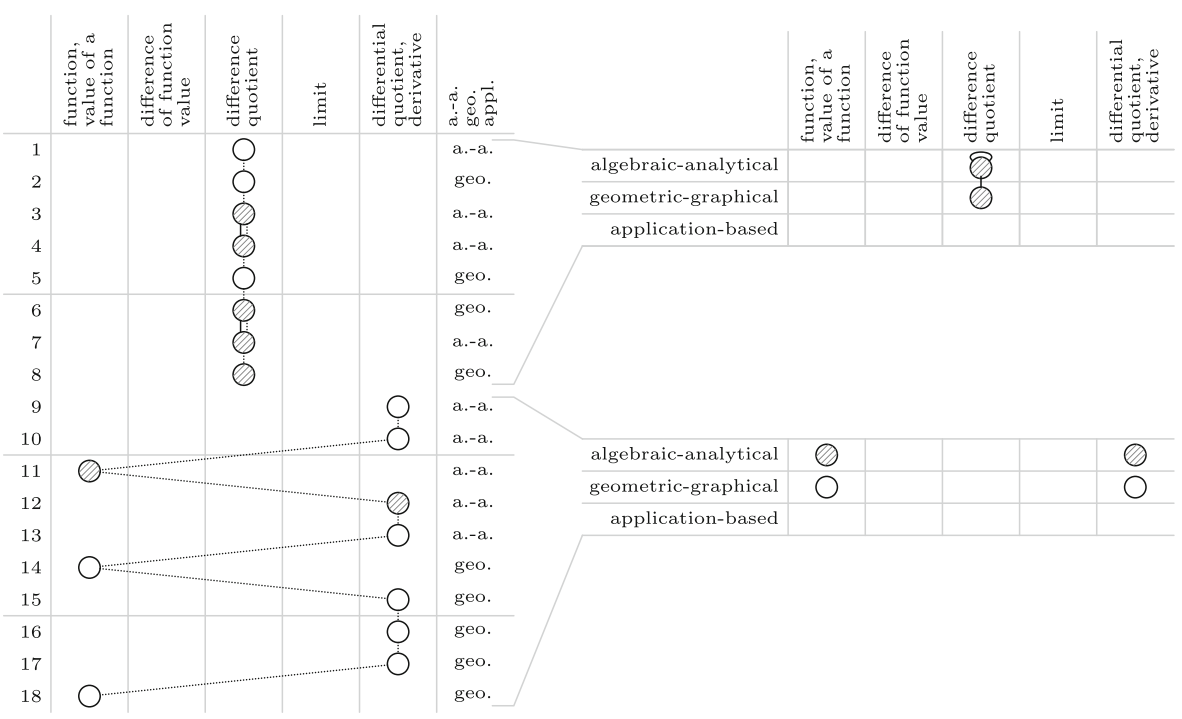

Fig. 6 Peter's imaginary dialogue (with a little error concerning where to draw $x_{0}$ and $x_{1}$ into the graph, and some confusion between "law of tangents" and "tangents") and the analysis sheet and analysis tables for his imaginary dialogue 


\section{Katharina and Maria: Problems with Speaking About the Limit}

However, it cannot be said that the use of diagrams always leads to speaking correctly about denotations. This becomes particularly clear when students talk about the limit. In some imaginary dialogues, for example, it is not clear whether the students can distinguish between the 0 , to which $h$ approaches, and the limit of the difference quotient itself. For instance, Katharina, an average-achieving student, writes:

This $\frac{f(x)-f\left(x_{0}\right)}{x-x_{0}}$ (here $x$ approaches 0 ) is actually the same as $\frac{f\left(x_{0}+h\right)-f\left(x_{0}\right)}{h}$ (here $h$ approaches 0 ).

This means that the variable is approaching the limit, i.e. as close to 0 as possible.

Katharina writes " $x$ approaches 0 " instead of $x_{0}$, but this could be a typo because above, she writes $\lim _{x \rightarrow x_{0}}$ correctly. However, later in the text it is not clear what exactly Katharina means by the term "limit", although in her imaginary dialogue she nicely connects the difference quotient with the differential quotient with diagrammatic activity.

Similarly, in the imaginary dialogue of another student, Maria, it is not clear, what exactly she is referring to with the term "limit" when she writes: "The limit (in German "Limes") is equal to the limit (in German "Grenzwert"), i.e. the smallest value that $h$ may take so that it does not become $0 ., 12$

\section{Comparing the Analysis Sheets and Analysis Tables of the Entire Class}

\section{A Response to RQ 2: Movements on the Analysis Sheet}

In considering the analysis sheets of the entire class, different types of movements between the columns are evident:

- Walk: Small movements between columns can be considered as a walk on the analysis sheet. Jakob's imaginary dialogue is an example: Jakob reasons about specific steps within the process that leads to the derivative. Some of the "walking" students mainly speak about this "walk" with no or little diagram use (as can be seen in Fig. 7 on the left). Others, such as Jakob, use diagrams and operations on diagrams to a large extent.

- Jump: Larger movements between columns appear as jumps on the analysis sheet. Most jumps appear between the columns "function, value of function" and "differential quotient, derivative", others between the columns "difference quotient" and "differential quotient, derivative" without addressing the limit. Often these "jumpers" stay in one column for some time, where they vertically connect verbal terms, followed up by a jump to another column, where verbal terms

\footnotetext{
${ }^{12}$ Maria's original text in German is: "Der Limes ist gleich der Grenzwert, das heißt der kleinste Wert, den $h$ einnehmen darf, damit er nicht 0 wird."
} 


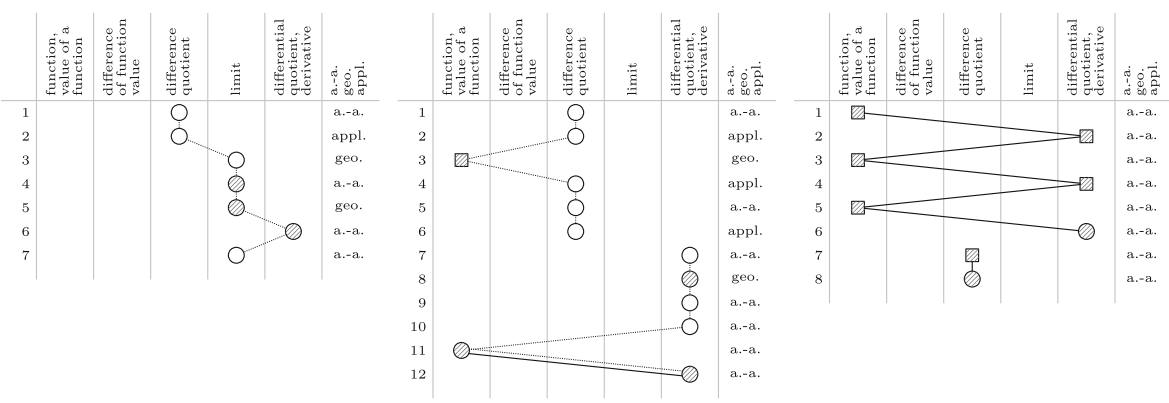

Fig. 7 The analysis sheets of three students

are linked. Examples can be seen in Oliver's analysis sheet and in Fig. 7 (in the middle).

- Split up: Finally, there are students who frequently split the line of thought, which is represented in the analysis sheets by unconnected circles. Examples can be seen in Fig. 7 (in the middle), and in Fig. 6. Of course, it is possible, that these students would be able to connect the different lines of thought if asked to. However, in my opinion, the very fact that they do not implement it in their imaginary dialogue shows a weaker connection than if they established the connections themselves.

\section{A Response to RQ 1: Differences Regarding the Analysis Table}

The analysis tables display the split ups as in the analysis sheet, here, as multiple tables for one student. They also display whether a student uses diagrams, which representational systems they belong to, algebraic-analytical or geometric-graphical, and whether the diagrams are connected by diagrammatic activity. Moreover, the use of application-based terms can be seen.

In the entire class, there is a wide range of different use of diagrams: Some students connect diagrams with denotations by writing diagrams without acting with them (see Fig. 8), some students connect diagrams vertically by explaining their correspondence in different representational systems (see Peter's imaginary dialogue and analysis tables), and some students use diagrammatic activity in order to horizontally connect diagrams and their denotations (see Jakob's analysis table).

\begin{tabular}{|c|c|c|c|c|c|}
\hline & 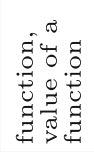 & 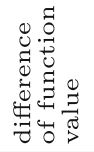 & 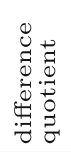 & 节 & 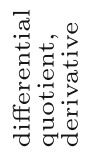 \\
\hline algebraic-analytical & & & 0 & 0 & $\oslash$ \\
\hline \multicolumn{6}{|l|}{ geometric-graphical } \\
\hline application-based & & & $\bigcirc$ & & $\bigcirc$ \\
\hline
\end{tabular}

Fig. 8 The analysis table of a student 
In particular, concerning the use of diagrammatic activity to horizontally link diagrams, there are those students who only link concrete functions with their derivative with the help of derivation rules and those who connect more "steps in between", like Jakob.

\section{Differences in Relation to the Mathematics Grade Achieved in the Previous Year}

Because of the small number of students, the differences listed here are only seen as an indication of how high-, average-, and low-achieving students may connect verbal terms and diagrams in this context. Of the total of 20 students, four students achieved a high grade for mathematics in the previous year's certificate, eleven achieved an average grade and six a low grade. These groups are referred to as high, average, and low achievers in what follows.

Concerning the movements in the analysis sheets (in relation to RQ 2), the highachieving students jump less often between columns and have fewer split ups in their line of thought. They rather make small movements between columns like a walk on the analysis sheet. In contrast, the average achievers jump more frequently and the low achievers even more frequently than the high achievers. Less frequently, a walking style can be found in these two groups. In addition, split ups are more common among the weaker students: None of the high achievers splits up their line of thought on paper. In contrast, almost $40 \%$ of the average achievers and a little over $80 \%$ of the low achievers have at least one split up.

Differences can also be found when looking at the use of diagrams and diagrammatic activity. The median of the ratio of shaded circles and squares to all circles and squares is $76 \%$ for the high achievers, $44 \%$ for the average achievers, and $38 \%$ for the low achievers. Differences in the average are about the same.

More distinct is the difference in the ratio of solid lines to all lines: the median for high achievers is $63 \%$, for average achievers $15 \%$, and for low achievers $8 \%$. Here, the average does not show such clear differences as the median: the ratio is $64 \%$ for high achievers, $18 \%$ for average achievers, but $29 \%$ for low achievers. The reason for the difference of the median and the average is the imaginary dialogue of one lowachieving student who uses diagrams and diagrammatic manipulations at every point (see Fig. 7 on the right) in contrast to the other low achievers. Thus, in terms of the occurrence of diagrams and diagrammatic activity there is no difference between this student and the higher achievers. In this case, however, it becomes clear how much the line of thought jumps back and forth between the column "function, value of a function" and "differential quotient, derivative". At this point it can be seen that one criterion such as the use of diagrams is not sufficient to clearly distinguish between the different groups.

However, overall, it can be said that the high achievers use diagrams more frequently, and above all, they act with diagrams considerably more frequently than the average achievers. Moreover, except for this one student, the same difference can be observed between average and low achievers.

Regarding the analysis table that focuses more on RQ 1, the clearest difference can be seen between the number of (diagrammatical) connections in the tables: The highachieving students establish an average of 4.3 connections, the average-achieving 
students 2.1 connections and the low-achieving students 1.2 connections. Altogether, most of the connections are horizontal, fewer are identity connections (i.e. when two diagrams of the same column are connected diagrammatically) and only a few are vertical.

With regard to the ratio of shaded to non-shaded circles or squares in the tables, there are hardly any differences between the three groups in the tables. The average of this ratio is $77 \%, 60 \%$, and $68 \%$ for the three groups, starting with the highachieving students (the median is: $80 \%, 67 \%$, and 68\%). Similarly, there is no distinct difference between the occurrence of algebraic-analytical, geometric-graphical and application-based terms or diagrams. Here, it is the case that the average- and lowachieving students speak a little more about geometric-graphical or application-based terms than the high-achieving students, who mainly argue algebraic-analytical.

\section{Discussion}

Previous works on learning differentiation have often concentrated on the students' conceptions (e.g. Zandieh (2000) findings about pseudo-objects). An additional perspective is provided by the approach presented here, which focuses on the students' sign activity and speaking about it as displayed in the students' writings. In this way, further effects on the learning process can be discussed that result from the differences observed in the students' lines of thought seen on paper, their diagrammatic activity, and how they speak about acting with diagrams.

\section{Consequences of the Way of Intertwining Diagram Activity and Speaking About It}

As seen above, the weaker students in particular acted with diagrams less frequently than the stronger students. This raises the question of what the consequences are for such students. In the framework outlined above, the meaning of a mathematical sign arises from its use within the sign game. Therefore, without sufficient activity with diagrams meaning cannot be grasped. One example can be found in Oliver's dialogue: since he does not act diagrammatically to get from $\frac{f\left(x_{0}+h\right)-f\left(x_{0}\right)}{h}$ to $\lim _{h \rightarrow 0} \frac{f\left(x_{0}+h\right)-f\left(x_{0}\right)}{h}$, in contrast to Jakob, Oliver can only speak about the limit superficially, saying: "That has something to do with the limit." Another example can be found in Peter's imaginary dialogue, when he compares diagrams without referring to their diagrammatical connections. Therefore, Peter cannot address their meaning, which arises precisely through the activity with diagrams.

Furthermore, the examples of Katharina's and Maria's problems with the limit showed that the use of diagrams does not always mean that the speaking about denotations will be correct. A possible explanation for difficulties with the limit could be the theoretical considerations that have been made above about denotations, interpretations, and diagrammatizations: As discussed previously, the step from $\frac{f(x+h)-f(x)}{h}$ to $\lim _{h \rightarrow 0} \frac{f(x+h)-f(x)}{h}$ requires a reflow from speaking about diagrams to diagrammatic activity, because of the phrase "for all $\varepsilon>0$ with $\varepsilon \in \mathbb{R}$ " in the definition 
of the limit of a function. Hence, one has to accept an interpretation in order to get to $\lim _{h \rightarrow 0} \frac{f(x+h)-f(x)}{h}$ and, in particular, it is not sufficient to only follow a line of diagrammatic activity.

This shows, how difficult it is for students to grasp the meaning of the limit. If one understands the meaning as its sign use, but the student's sign use is limited (for example if the limit is only used as a late substituting) and diagrammatizations of interpretations are included, the meaning cannot be fully comprehended. The difficulty for students here lies within the complex intertwining of diagrammatical activity and speaking about it.

\section{Conclusion}

From the viewpoint of a framework based on Wittgenstein and Peirce where diagram activity and speaking about it are both inevitable and central to mathematical activity, the intertwining of the two becomes the focus. For a student, in order to progressively learn about the meaning of mathematical diagrams and their denotations it means that he or she needs numerous experiences both in diagram activity and speaking about it.

The analyzing method presented in this article can be a tool to display this intertwining. For the topic of "derivative", regarding RQ 1, it became apparent from the analysis tables which connections are actually made by speaking about diagram activity and which by the diagram activity itself. Additionally, regarding RQ 2, the different types of movement, walk, jump, and split up became apparent with the help of the analysis sheets.

In the light of the framework presented here, understanding mathematics means that a student learns more and more about the rules of the "game" and how they can be derived from other rules, thus, how diagrams and propositions about diagrams are connected. This requires both diagram activity and speaking about diagram activity. Furthermore, a student can better comprehend the meaning of a diagram the more experience he or she has with extensive diagrammatical use, possibly in different representational systems. In particular, speaking about diagrams is needed to formulate properties and theorems, but it cannot replace diagrammatic activity and there is a risk that speaking about diagrams might be detached from the diagram activity itself. In cases that include diagrammatizations of interpretations, a student's diagram activity and his or her speaking about this activity should be taken into consideration when evaluating the student's mathematical understanding. In the examples outlined here this concerned the limit.

What does this mean in terms of the findings of the case study? It was shown that the lower achievers in particular acted diagrammatically less frequently and when they did, they mainly used derivation rules without the steps in between, specifically, they jumped more often or had more split ups. This means, in order to support these students, it is not enough to show them horizontal and vertical connections only by talking about them. Instead, they must be offered a variety of learning opportunities where they can both be diagrammatically active and express this in language. In particular, not only should connections between a function and its derivative be diagrammatically established, but also the "steps in between". 
Furthermore, in order to study the students' understanding both how they speak about the denotations and how they act diagrammatically are worth investigating, especially in cases where diagrammatizations of interpretations are involved. The method presented here is one way in which this could be done.

Funding Information Open access funding provided by University of Klagenfurt.

Open Access This article is distributed under the terms of the Creative Commons Attribution 4.0 International License (http://creativecommons.org/licenses/by/4.0/), which permits unrestricted use, distribution, and reproduction in any medium, provided you give appropriate credit to the original author(s) and the source, provide a link to the Creative Commons license, and indicate if changes were made.

\section{References}

Danckwerts, R., \& Vogel, D. (2006). Analysis verständlich unterrichten [Teaching analysis in a comprehensible way]. München: Spektrum Akademischer Verlag.

Dörfler, W. (2005). Diagrammatic thinking. Affordances and constraints. In Hoffmann, M.H.G., Lenhard, J., Seeger, F. (Eds.) Activity and sign. Grounding Mathematics Education (pp. 57-66). New York, NY: Springer.

Dörfler, W. (2006). Diagramme und Mathematikunterricht [Diagrams and teaching mathematics]. Journal für Mathematik-Didaktik, 27(3-4), 200-219. https://doi.org/10.1007/BF03339039.

Dörfler, W. (2008). Mathematical reasoning: Mental activity or practice with diagrams. In Niss, M. (Ed.) ICME 10 Proceedings, Regular Lectures, CD-Rom (p. 17). Roskilde, Denmark: Roskilde University and IMFUFA.

Dörfler, W. (2016). Signs and their use: Peirce and Wittgenstein. In Bikner-Ahsbahs, A., Vohns, A., Schmitt, O., Bruder, R., Dörfler, W. (Eds.) Theories in and of Mathematics Education. Theory Strands in German Speaking Countries (ICME 13 Topical Surveys) (pp. 21-31). Berlin: Springer.

Duval, R. (2006). A cognitive analysis of problems of comprehension in the learning of mathematics. Educational Studies in Mathematics, 61(1-2), 103-131. https://doi.org/10.1007/s10649-006-0400-z.

Eisenman, P. (2008). Why is it not true that $0.999 \ldots<1$ ? The Teaching of Mathematics, 11(1), 35-40.

Ferrini-Mundy, J., \& Graham, K. (1991). An overview of the calculus curriculum reform effort: Issues for learning, teaching, and curriculum development. American Mathematical Monthly, 98(7), 627-636. https://doi.org/10.1080/00029890.1991.11995769.

Gray, E., \& Tall, D. O. (1994). Duality, ambiguity, and flexibility: A "Proceptual" view of simple arithmetic. Journal for Research in Mathematics Education, 25(2), 116-140. https://doi.org/10.2307/ 749505.

Hoffmann, M. H. G. (2005a). Erkenntnisentwicklung. Ein semiotisch-pragmatischer Ansatz [Development of knowledge A semiotic-pragmatic approach]. Frankfurt am Main: Vittorio Klostermann $\mathrm{GmbH}$.

Hoffmann, M. H. G. (2005b). Signs as means for discovery. In Hoffmann, M.H.G., Lenhard, J., Seeger, F. (Eds.) Activity and sign. Grounding mathematics education (pp. 45-56). New York, NY: Springer.

Hoffmann, M. H. G. (2007). Cognitive conditions of diagrammatic reasoning (Georgia Tech's School of Public Policy Working Paper Series 24). Retrieved from http://works.bepress.com/michael_hoffmann/1/.

Koch, P., \& Österreicher, W. (2012). Language of immediacy - language of distance: Orality and literacy from the perspective of language theory and linguistic history. In Lange, C., Weber, B., Wolf, G. (Eds.) Communicative Spaces. Variation, Contact, and Change- Papers in Honour of Ursula Schaefer (pp. 441-473). Bern: Peter Lang.

Monaghan, J. (2001). Young peoples' ideas of infinity. Educational Studies in Mathematics, 48, $239-257$. https://doi.org/10.1023/A:1016090925967.

Müller-Hill, E., \& Wille, A. M. (2018). Negotiating mathematical meaning with oneself - snapshots from imaginary dialogues on recurring decimals. In Norén, E., Palmér, H., Cooke, A. (Eds.) Skrifter från Svensk Förening för MatematikDidaktisk Forskning, No. 12, Nordic Research in Mathematics Education (pp. 69-77). Göteborg, Sweden: SMDF. 
Orton, A. A. (1983). Students' understanding of differentiation. Educational Studies in Mathematics, 14(3), 235-250. https://doi.org/10.1007/BF00410540.

Otte, M. F. (2011). Evolution, learning, and semiotics from a Percean point of view. Educational Studies in Mathematics, 77, 313-329. https://doi.org/10.1007/s10649-011-9302-9.

Park, J. (2015). Is the derivative a function? If so, how do we teach it? Educational Studies in Mathematics, 89, 233-250. https://doi.org/10.1007/s10649-015-9601-7.

Peirce, (NEM). (1976). The New Elements of Mathematics by Charles S. Peirce. In Eisele, C. (Ed.), Vol. I-809 IV. The Hague-Paris/Atlantic Highlands, N.J.: Mouton/Humanities Press.

Rasmussen, C., Marrongelle, K., Borba, M.C. (2014). Research on calculus: what do we know and where do we need to go?. ZDM Mathematics Education, 46, 507-515. https://doi.org/10.1007/s11858014-0615-X.

Staats, S. K. (2008). Poetic lines in mathematics discourse: A method from linguistic anthropology. For the Learning of Mathematics, 28(2), 26-32.

Tall, D. O., \& Schwarzenberger, L. E. (1978). Conflicts in the learning of real numbers and limits. Mathematics Teaching, 82, 44-49.

Thompson, P. W. (1994a). Images of rate and operational understanding of the Fundamental Theorem of Calculus. Educational Studies in Mathematics, 26(2-3), 229-274. https://doi.org/10.1007/ BF01273664.

Thompson, P. W. (1994b). Students, functions, and the undergraduate curriculum. In Dubinsky, E., Schoenfeld, A.H., Kaput, J.J. (Eds.) Research in collegiate mathematics education, 1, Issues in Mathematics Education, (Vol. 4 pp. 21-44). Providence, RI: American Mathematical Society.

Vygotsky, L. S. (1986). Thought and language (A. Kozulin, Trans. and Ed.) Cambridge: M.I.T. Press.

Weigand, H.-G. (2014). A discrete approach to the concept of derivative. ZDM Mathematics Education, 46, 603-619. https://doi.org/10.1007/s11858-014-0595-x.

Wille, A. M. (2008). Aspects of the concept of a variable in imaginary dialogues written by students. In Figueras, O., Cortina, J., Alatorre, S., Rojano, T., Sepúlveda, A. (Eds.) Proceedings of the 32nd Conference of the International Group for the Psychology of Mathematics Education (PME32), (Vol. 4 pp. 417-424). Mexico: CINVESTAV and PME.

Wille, A. M. (2017a). Imaginary Dialogues in Mathematics Education. Journal für Mathematik-Didaktik, 38(1), 29-55. https://doi.org/10.1007/s13138-016-0111-7.

Wille, A. M. (2017b). Conceptions of the transition from the difference quotient to the derivative in imaginary dialogues written by preservice teachers. In Dooley, T., \& Gueudet, G. (Eds.) Proceedings of the Tenth Congress of the European Society for Research in Mathematics Education (CERME10, February 1 - 5, 2017, pp. 1396-1403). Dublin, Ireland: DCU Institute of Education and ERME.

Wittgenstein, L. (1999). (PI). Philosophical investigations (second edition) (G. E. M. Anscombe, Trans.) Oxford/Malden: Blackwell Publishers Ltd.

Wittgenstein, L. (1967). (RFM). Remarks on the foundation of mathematics. (G. H. Wright, R. Rhees, G. E. M. Anscombe, Eds.) (G. E. M. Anscombe, Trans.) Cambridge: M.I.T. Press.

Wittgenstein, L. (1979). Wittgenstein and the Vienna Circle: Conversations recorded by F. Waismann. In MacGuiness, B. (Ed.) New York: Barnes \& Noble.

Wrigley, M. (1977). Wittgenstein's philosophy of mathematics. The Philosophical Quarterly (1950-), 27(106), 50-59. https://doi.org/10.2307/2218928.

Zandieh, M. J. (2000). A theoretical framework for analyzing student understanding of the concept of derivative. CBMS Issues in Mathematics Education, 8, 103-127.

Zazkis, R., Liljedahl, P., Sinclair, N. (2013). Lesson play in mathematics education. A tool for research and professional development. New York, NY: Springer.

Publisher's Note Springer Nature remains neutral with regard to jurisdictional claims in published maps and institutional affiliations. 